\title{
Localización efectiva de un segundo conducto radicular en incisivos inferiores mediante magnificación, radiografía diafanización.
}

\section{Effective location a second root canal in lower incisors by magnification radiography and diaphanization}

Thais Mageste Duque ${ }^{1, a}$, Daniel Rodrigo Herrera Morante ${ }^{1, a}$, Caio Cézar Randi Ferraz ${ }^{1, b}$, Alexandre Augusto Zaia ${ }^{1, b}$, José Flávio Affonso de Almeida ${ }^{1, b}$, Brenda Paula Figueiredo de Almeida Gomes ${ }^{1, c}$

\section{RESUMEN}

Objetivo: Evaluar in vitro, la efectividad del método visual y radiográfico, comparándolos con la técnica de diafanización en la detección del segundo conducto radicular de incisivos inferiores tratados endodónticamente. Material y métodos: Fueron analizados 133 dientes incisivos inferiores permanentes, tratados endodónticamente, a través de métodos visuales (con y sin auxilio de magnificación), radiográficos y de diafanización. El análisis radiográfico fue realizado en negatoscopio utilizando una lupa estereoscópica (10X). Posteriormente, fue realizada la remoción completa de la obturación provisional para la exploración visual con y sin auxilio de microscopio clínico (MO) en un aumento de 12X. Finalmente los dientes fueron diafanizados y almacenados en salicilato de metila para posterior análisis. Dos evaluadores calibrados registraron los datos referentes a la presencia o no del segundo conducto radicular para cada uno de los métodos utilizados. Resultados: El análisis radiográfico mostró la presencia del segundo conducto en 24 dientes $(18,05 \%)$. Visualmente, fue encontrado en 4 dientes $(3,00 \%)$ sin magnificación y en 16 dientes (12,03\%) con ayuda del MO. A través de la diafanización, 34 dientes $(25,95 \%)$ presentaron segundo conducto. Conclusiones: La asociación de técnicas radiográficas y la magnificación visual obtenida con la utilización del MO se muestran como los aliados clínicos más efectivos para la localización del segundo conducto radicular en incisivos inferiores.

\section{Palabras clave: ENDODONCIA / CAVIDAD PULPAR / ANATOMÍA.}

\author{
ABSTRACT \\ Objective: To evaluate in vitro the effectiveness of the visual, radiographic and clearing technique on detection of two root \\ canals in mandibular incisor teeth endodontically treated. Material and methods: One hundred thirty-three human per- \\ manent lower incisors were analyzed through visual methods (with and without magnification), radiographic and clearing \\ technique. Radiographic analysis was performed in light box using a stereomicroscope (10X). Then, the temporary filling of \\ al specimens was removed for visual exploration with and without clinical microscope (OM) (12X). Finally the teeth were \\ ${ }_{1}$ Departamento de Odontología Restauradora, Área de Endodoncia. Facultad de Odontología de Piracicaba, Universidad \\ Estadual de Campinas (FOP-UNICAMP). Sau Paulo, Brasil. \\ a Doctorando en Clínica Odontológica, Área de Endodoncia \\ b Profesor Asociado \\ c Profesor Titular
}


submitted to clearing technique and stored in methyl salicylate for subsequent analysis. Two calibrated reviewers recorded data about the presence or absence of the second root canal for each of the used methods. Results: Radiographic analysis showed the presence of the second root canal in 24 teeth (18.05\%). Visually, it was found in 4 teeth (3.00\%) and 16 teeth $(12.03 \%)$ without and with the use of OM, respectively. Through clearing technique, in 34 teeth (25.95\%) it was found the second root canal. Conclusion: The association of radiographic techniques and visual magnification obtained with the use of MO has been shown as the most effective clinical partners to locate the second root canal in mandibular incisors.

\section{Key words: ENDODONTICS / DENTAL PULP CAVITY / ANATOMY}

\section{INTRODUCCIÓN}

El éxito del tratamiento endodóntico está relacionado directamente con el conocimiento de la anatomía interna del diente por el operador (1).

Dentro de los diferentes grupos de diente, los incisivos inferiores se presentan como un desafío debido al tamaño y anatomía variada (2). En términos generales estos dientes son considerados como unirradiculares, de conducto ancho en sentido vestíbulolingual y estrecho en sentido mesiodistal (3). En algunos casos, debido al acentuado achatamiento mesiodistal, pueden presentar bifurcaciones en el conducto, pero que terminan en un único foramen. Raramente esta bifurcación es completa, terminando en dos forámenes apicales distintos (4). La no localización de este segundo canal ocurre en 40\% de los casos (5). Según Gomes et al, el $64 \%$ de los incisivos inferiores presentan conducto único y el 36\% múltiples conductos (6).

Una evaluación radiográfica preoperatoria minuciosa es necesaria e indispensable para el éxito de la terapia endodóntica (7). El examen radiográfico asociado al conocimiento de la anatomía, pueden favorecer la visualización de una imagen tridimensional, principalmente en incisivos inferiores (4), sin embargo, raramente muestran la posible presencia de un segundo conducto (8).

Hoy en día el microscopio óptico (MO) es un aliado en el tratamiento endodóntico, permitiendo una visualización más precisa y con mayor riqueza los detalles (9). La localización de los conductos radiculares se ve facilitada en gran medida con el uso del MO cuando se compara al uso de lupas o a la simple visualización a ojo desnudo (10)

La diafanización es un método práctico, fácil, económico y de gran utilidad para el estudio de la anatomía interna de los dientes. Esta técnica permite el estudio tridimensional de la anatomía, siendo más preciso que el análisis radiográfico (11) y pudiendo servir como control positivo en estudios in vitro.

De esta manera, el presente estudio tuvo por objetivo evaluar in vitro, la efectividad del método visual y radiográfico, comparándolos con la técnica de diafanización en la detección del segundo conducto radicular de incisivos inferiores tratados endodónticamente.

\section{MATERIAL Y MÉTODOS}

El estudio fue aprobado por el Comité de Ética en Pesquisa de la Facultad de Odontología de Piracicaba, Universidad Estadual de Campinas (FOP-UNICAMP) bajo el protocolo 148/2010. Fueron analizados 133 incisivos inferiores permanentes humanos. Estos dientes fueron tratados endodónticamente en la disciplina de preclínica como parte del entrenamiento de laboratorio para alumnos de pregrado de la FOP-UNICAMP.

\section{Evaluación radiográfica}

Todos los dientes fueron radiografiados tanto en sentido orto-radial como mesio-distal utilizando películas radiográficas Ultra-speed (Eastman Kodak Company, Rochester, NY, EEUU) con parámetros de exposición de $60 \mathrm{kVp}$ y $10 \mathrm{~mA}$ a una distancia de $15 \mathrm{~cm}$ y un tiempo de exposición $0.54 \mathrm{~s}$ (Gnatus XR 6010, Ribeirão Preto, SP, Brasil).

El análisis radiográfico fue realizado en negatoscopio utilizando una lupa estereoscópica (10X). Dos evaluadores calibrados registraron informaciones referentes al número de conductos existentes y la condición de la obturación. Del mismo modo, cada diente fue identificado según la clasificación de Vertucci (1) (Cuadro 1).

\section{Evaluación visual}

Fue removida la restauración provisoria de todos los dientes utilizando fresas diamantadas, permitiendo así la visualización directa del material obturador. 


\begin{tabular}{|c|c|}
\hline Tipo & Descripción \\
\hline I & Diente con un único conducto desde la cámara pulpar hasta el ápice. \\
\hline II & $\begin{array}{l}\text { Diente con dos conductos separados que dejan la cámara pulpar y se unen cerca del ápice terminando en } \\
\text { un único conducto. }\end{array}$ \\
\hline III & $\begin{array}{l}\text { Diente con un único conducto que deja la cámara pulpar, se divide en dos y luego se unen terminando en } \\
\text { un único conducto. }\end{array}$ \\
\hline IV & Diente con dos conductos separados y distintos desde la cámara pulpar hasta el ápice. \\
\hline $\mathrm{V}$ & $\begin{array}{l}\text { Diente con un conducto que deja la cámara pulpar, se divide en dos cerca del ápice que terminan en } \\
\text { forámenes separados. }\end{array}$ \\
\hline VI & $\begin{array}{l}\text { Diente con dos conductos separados en la cámara pulpar que se unen en } \\
\text { el cuerpo de la raíz y se dividen nuevamente cerca del ápice terminando en forámenes distintos. }\end{array}$ \\
\hline VII & $\begin{array}{l}\text { Diente con un conducto que deja la cámara pulpar, se divide en dos conductos, que después se unen y } \\
\text { vuelven a separarse en dos conductos. }\end{array}$ \\
\hline VIII & Diente con tres conductos separados desde la cámara pulpar hasta el ápice. \\
\hline
\end{tabular}

* Adaptado de Vertucci FJ (1).

Utilizando limas K \#10 y explorador endodóntico se intentó localizar la existencia de un posible segundo conducto.

El mismo análisis fue realizado también con ayuda del MO (Opto DM Plus-São Paulo, Brasil) en un aumento de 12X. Datos referentes al número de conductos obturados y existentes fueron registrados por los evaluadores tanto para la visualización a ojo desnudo como para el análisis con MO.

Después de esta evaluación los dientes fueron cortados transversalmente a nivel de la unión cemento-adamantina e analizados en lupa estereoscópica (Leica MZ75, Leica Microsystems GmbH, Wetzlar, Alemania) (2X) con la finalidad de capturar imágenes representativas de los datos obtenidos.
Para la diafanización, la superficie externa de los especímenes fue impermeabilizada con esmalte para uña (Colorama, Bozzano Ceil, SP, Brasil). A continuación, fueron inmersos en tinta china (Staedtler, Nuremberg, Alemania) y sometidos a bomba de vacío (TECNAL TE-058, Piracicaba, SP, Brasil) por 1 h / $700 \mathrm{mmHg}$, luego de lo cual permanecieron en la tinta por 7 días a $37^{\circ} \mathrm{C}$. Pasado este periodo los especímenes fueron lavados en agua corriente por $24 \mathrm{~h}$ para remover el exceso de tinta.

En seguida fue realizada la descalcificación de los especímenes con ácido clorhídrico al 5\% por un periodo de 3-6 días dependiendo del grado de descalcificación de cada uno. Los especímenes fueron lavados en agua corriente por $4 \mathrm{~h}$ para la remoción total del ácido. En ese momento los especímenes

Tabla 1. Número y frecuencia (\%) de incisivos inferiores posibles de ser clasificados según la morfología del sistema de conductos radiculares* con los diferentes métodos evaluados.

\begin{tabular}{lcccccccc}
\hline \multicolumn{7}{c}{ Clasificación* } \\
\hline Método & I & II & III & IV & V & VI & VII & VIII \\
& & & & 1 & & & \\
RX & $109(81,95 \%)$ & $7(5,26 \%)$ & $16(12,03 \%)$ & 0 & $(0,75 \%)$ & 0 & 0 & 0 \\
VSMO & 0 & 0 & 0 & 0 & 0 & 0 & 0 & 0 \\
VCMO & 0 & $16(12,03 \%)$ & 0 & 0 & 0 & 0 & 0 & 0 \\
& & & & & 2 & & & 0 \\
TD & $97(74,05 \%)$ & $19(14,50)$ & $13(9,92 \%)$ & 0 & $(1,53 \%)$ & 0 & 0 & 0 \\
\hline
\end{tabular}




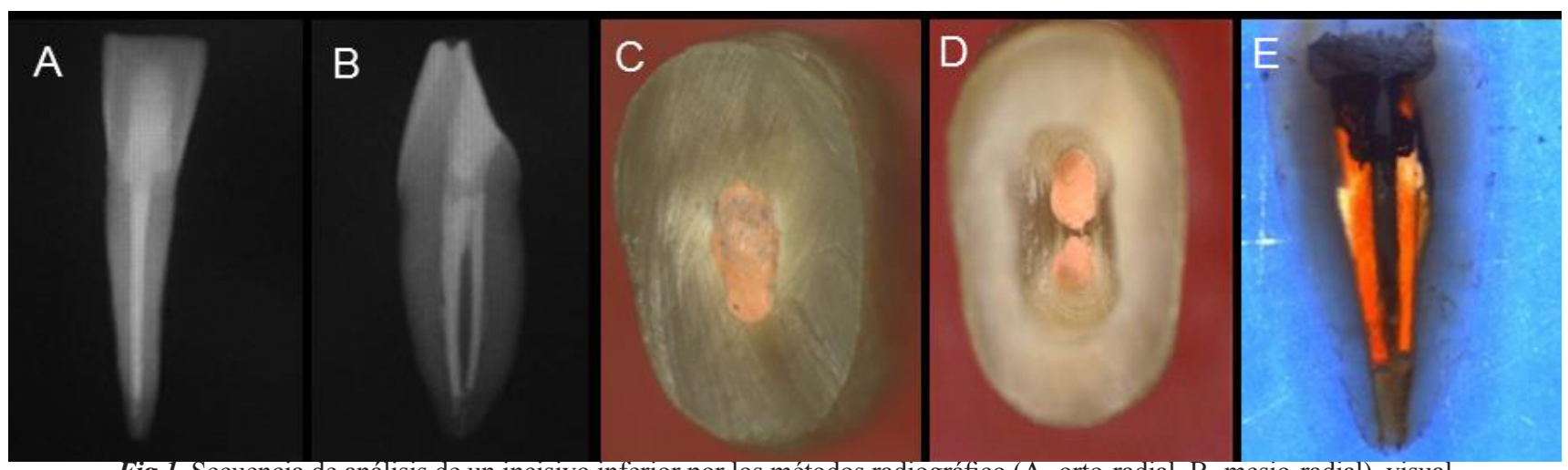

Fig 1. Secuencia de análisis de un incisivo inferior por los métodos radiográfico (A- orto-radial, B- mesio-radial), visual

(C- inmediatamente después del corte; D- después de la remoción de gutapercha del tercio cervical) y por diafanización (E).

pasaron por un proceso de deshidratación progresiva a través de escala ascendente de alcohol $\left(70^{\circ}, 80^{\circ}\right.$, $85^{\circ}, 90^{\circ}, 95^{\circ}$ y $\left.100^{\circ} \mathrm{GL}\right)$, permaneciendo $2 \mathrm{~h}$ en cada concentración, siendo que para el alcohol absoluto, fueron realizadas dos renovaciones a cada $3 \mathrm{~h}$.

Finalmente, los especímenes fueron inmersos individualmente e tubos tipo eppendorf, conteniendo una solución de salicilato de metila PA para obtener la transparencia deseada y al mismo tiempo almacenarlos para posterior evaluación. Con los especímenes ya diafanizados fueron capturadas imágenes a través del estereomicroscópio para el análisis en detalle de la anatomía interna.

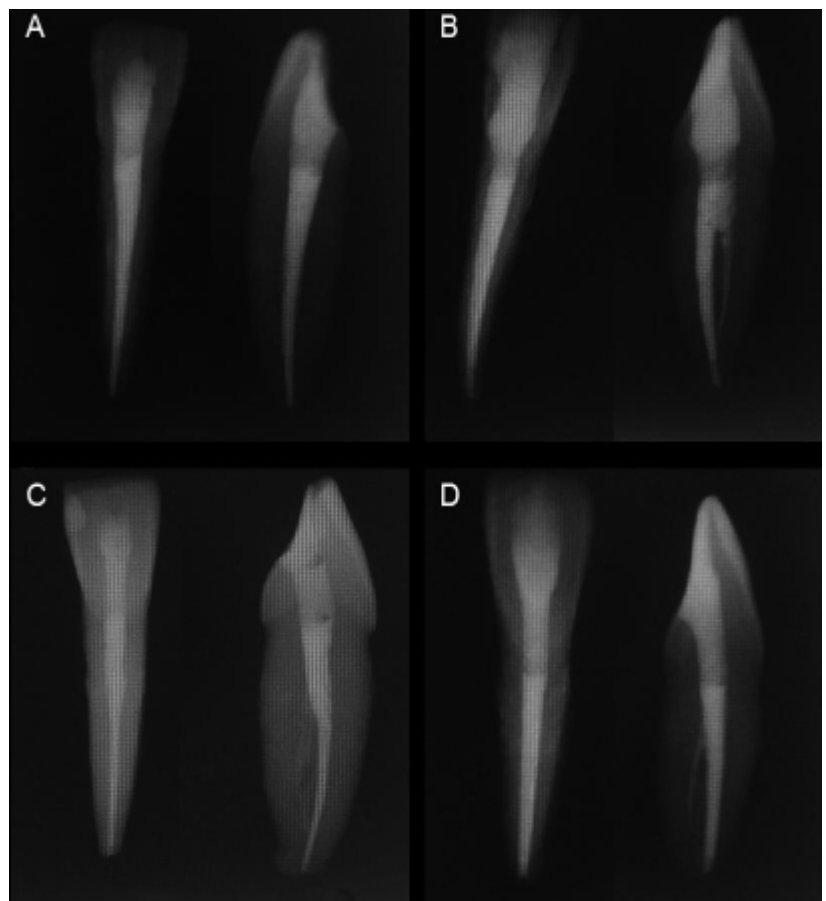

Fig 2. Sistema de conductos Tipo I (A), Tipo II (B), Tipo III (C) y Tipo $V(D)$ evaluados por método radiográfico.

\section{RESULTADOS}

Los resultados obtenidos muestran en cada tipo de evaluación siguiendo los criterios de la clasificación de Vertucci (Tabla 1). Puede observarse que únicamente el método radiográfico y la técnica de diafanización permitieron la clasificación de todos los dientes evaluados. Por otro lado, la Tabla 2 muestra la cantidad de incisivos inferiores que presentaron dos conductos radiculares después de evaluados por los diferentes métodos. La Fig 1. Muestra la secuencia del análisis en un mismo diente.

\section{Evaluación radiográfica}

A través de la evaluación radiográfica, únicamente

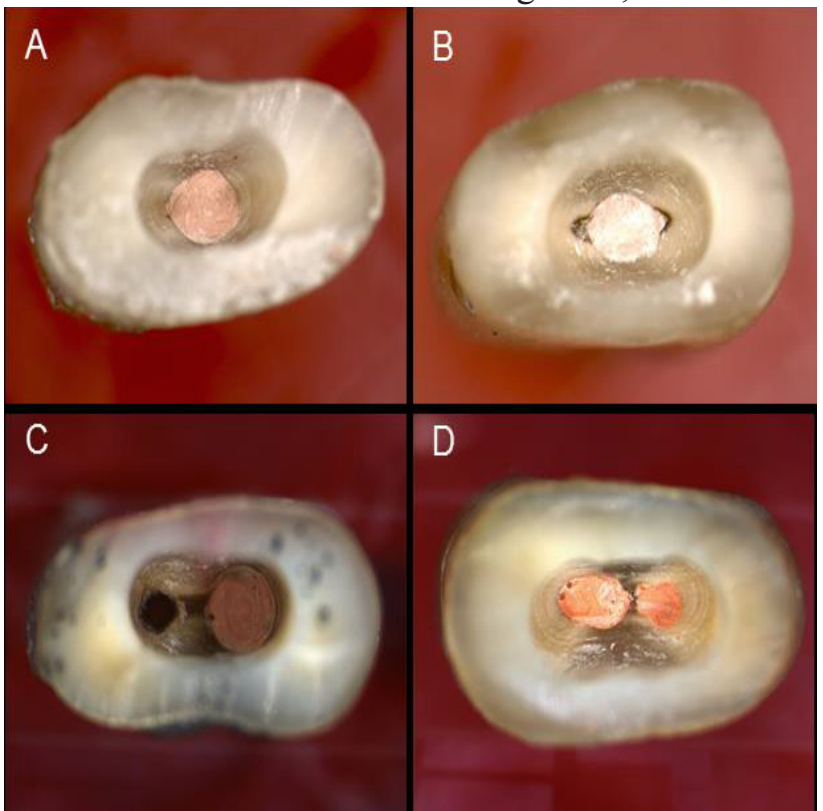

Fig 3. Diferentes tipos de conducto radicular posibles de ser observados clínicamente. (A) conducto radicular único y centralizado; (B) conducto radicular e istmos bilaterales; (C) un conducto radicular obturado y uno sin obturar; (D) dos conductos radiculares obturados. Imágenes obtenidas en lupa estereoscópica $(2 X)$. 
4 dientes $(3,0 \%)$ presentaron 2 conductos radiculares instrumentados y obturados. Otros 20 dientes $(15,04 \%)$ presentaron una imagen sugestiva de presencia de un segundo conducto. La radiografía mesio-distal evidenció de manera más clara la presencia del segundo conducto.

En la Fig 2. Se presentan imágenes representativas de los diferentes tipos de conducto radicular encontrados durante la evaluación radiográfica.

\section{Evaluación visual}

En la evaluación clínica visual, donde fue utilizada únicamente la luz del reflector, 4 dientes $(3,00 \%)$ presentaron segundo conducto. Por otro lado, fueron encontrados 50 dientes $(37,60 \%)$ con conducto radicular asociado a la presencia de istmos.

En la evaluación visual con uso de MO fueron encontrados 39 dientes $(29,32 \%)$ con conducto radicular asociado a la presencia de istmos.

De los 133 incisivos evaluados, 16 (12,03\%) presentaron 2 conductos radiculares que terminaban apenas en un foramen radicular. De esos casos, $11(68,75 \%)$ eran incisivos laterales inferiores y 5 $(31,25 \%)$ eran incisivos centrales inferiores.

En la Fig 3. Se presentan imágenes representativas de los diferentes tipos de conducto radicular observados clínicamente.

\section{Diafanización}

Durante el proceso de diafanización 2 dientes fueron perdidos. De los 131 dientes finalmente evaluados, 34 $(25,96 \%)$ presentaron segundo conducto radicular.

En la Fig 4. Se presentan imágenes representativas de los diferentes tipos de conducto radicular encontrados por el método de diafanización.

\section{DISCUSIÓN}

En general el incisivo inferior es tratado por el clínico como un diente unirradicular. Sin embargo, resultados de estudios previos, así como los obtenidos en este estudio muestran que este grupo dentario puede presentar 2 conductos radiculares.

Considerando la evaluación radiográfica, nuestros resultados mostraron el $18,05 \%$ de los incisivos
Tabla 2. Número y frecuencia (\%) de incisivos inferioresque presentaron dos conductos radiculares despúes de evaluados por diferentes métodos.

\begin{tabular}{lr}
\hline Método & Número y Frecuencia \\
\hline RX & $24(18,05 \%)$ \\
VSMO & $4(3,00 \%)$ \\
VCMO & $16(12,03 \%)$ \\
TD & $34(25,95 \%)$ \\
\hline
\end{tabular}

RX-Método radiográfico; VSMO- Método visual sin microscópio

clínico; VCMO- Método visual con microscópio clínico; TD-Técnica de diafanización.

inferiores con 2 conductos radiculares. Resultado que concuerdan con la literatura, donde esta tasa puede variar en rangos que van desde el $20 \%$ hasta $45 \%(1,3,11,13)$. Los conductos radiculares correspondientes al Tipo I totalizaron el $81,90 \%$ de la muestra, resultados comparables con los obtenidos por Miyashita et al. (14). Sin embargo, no se encontraron conductos radiculares correspondientes al Tipo IV.

A pesar de que clínicamente sea viable apenas la obtención de radiografías orto-radiales o ligeramente disociadas, nuestros resultados muestran que la radiografía mesio-radial facilita el análisis de la real anatomía interna del conducto radicular. Así, el intentar la disociación es una opción clínica importante y factible.

Durante el tratamiento endodóntico de incisivos inferiores, la visión directa es limitada al orificio de entrada del conducto, lo que dificulta la localización de un posible segundo conducto. Esto se confirma con nuestros resultados, donde apenas el 3,00\% de la muestra presentó un segundo conducto radicular.

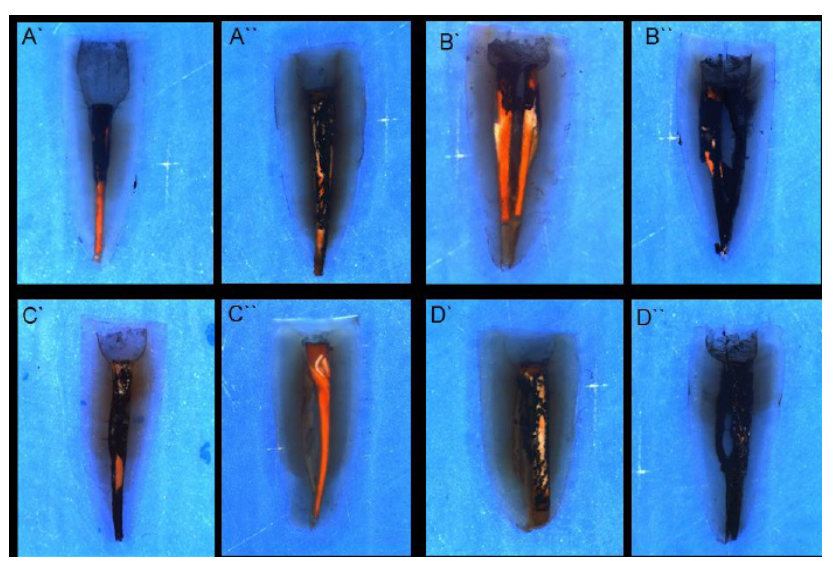

Fig 4. Sistema de conductos Tipo I (A'y A'), Tipo II (B'y B'), Tipo III (C'y C') y Tipo V (D'y D') evaluados por la técnica de diafanización. 
El uso del magnificación asociada a iluminación, favorece la visualización de la entrada de los conductos radiculares. Sin embargo, en este estudio los dientes ya presentaban tratamiento endodóntico, y esta obturación puede limitar la visualización. Esto se confirma con nuestros resultados, donde el análisis radiográfico fue mejor para identificar el segundo conducto radicular comparado a la evaluación con MO.

La evaluación con $\mathrm{MO}$ detecto visualmente la presencia de un segundo conducto radicular en 3,0\% de los incisivos centrales y $16,75 \%$ de los incisivos laterales, resultados que concuerdan con los obtenidos por Yoshioka et al. (10).

Los resultados obtenidos en la diafanización son semejantes a los descritos en la literatura $(6,11)$ y es válido utilizar ellos como nuestro control positivo. Así podemos decir que la técnica radiográfica permitió localizar el 70,59\% de los dientes que tenían 2 conductos radiculares, la evaluación visual sin MO únicamente el 11,76\% y la evaluación con MO el 47,05\%.

Con base en la metodología utilizada en el presente estudio y los resultados obtenidos, podemos resaltar la importancia de un análisis clínico y radiográfico minucioso que permita al clínico descartar la presencia de un segundo conducto radicular en incisivos inferiores, situación en la cual el MO juega un papel importante.

\section{CONCLUSIONES}

La asociación de técnicas radiográficas y la magnificación visual obtenida con la utilización del MO se muestran como los aliados clínicos más efectivos para la localización del segundo conducto radicular en incisivos inferiores.

\section{CORRESPONDENCIA}

\section{Brenda P. F. A. Gomes}

Av. Limeira 901, Areão, Piracicaba. São Paulo, Brasil e-mail: bpgomes@fop.unicamp.br

\section{REFERENCIAS BIBLIOGRÁFICAS}

1. Vertucci FJ. Root canal anatomy of the human permanent teeth. Oral Surg Oral Med Oral Pathol. 1984;58(5):589-99.

2. Mauger MJ, Waite RW, Alexander JB, Schindler WG. Ideal endodontic access in mandibular incisors. J Endod. 1999;25(3):206-7.

3. Benjamin KA, Dowson J. Incidence of two root canals in human mandibular incisor teeth. Oral Surg Oral Med Oral Pathol. 1974;38(1):122-6.

4. Loushine RJ, Jurcak JJ, Jeffalone DM. A two-rooted mandibular incisor. J Endod. 1993;19(5):250-1.

5. Martos J, Lubian C, Silveira LF, Castro LA, Luque CM. Morphology analysis of the root apex in human teeth. J Endod 2010;36(4):664-7.

6. Gomes BP, Rodrigues HH, Tancredo N. The use of a modelling technique to investigate the root canal morphology of mandibular incisors. Int Endod $\mathrm{J}$. 1996;29(1):29-36.

7. Tiku AM, Kalaskar RR, Damle SG. An unusual presentation of all the mandibular anterior teeth with two root canals. A case report. J Indian Soc Pedod Prev Dent. 2005;23(4):201-6.

8. Warren EM, Laws AJ. The relationship between crown size and the incidence of bifid root canals in mandibular incisor teeth. Oral Surg Oral Med Oral Pathol. 1981;52(4):425-9.

9. Buhrley LJ, Barrows MJ, BeGole EA. Effect of magnification on locating the MB2 canal in maxillary molars. J Endod. 2002;28(4):324-6.

10. Yoshioka T, Kobayashi C, Suda H. Detection rate of root canal orifices with a microscope. J Endod. 2002;28(6):452-3.

11. Kartal N, Yanikoglu FC. Root canal morphology of mandibular incisors. J Endod. 1992;18(11):562-4.

12. Madeira MC, Hetem S. Incidence of bifurcations in mandibular incisors. Oral Surg Oral Med Oral Pathol. 1973;36(4):589-91.

13. Green D. A Stereomicroscopic study of the root apices of 400 maxillary and mandibular anterior teeth. Oral Surg Oral Med Oral Pathol.1956;9(1):1224-8.

14. Miyashita M, Kasahara E. Root Canal System of the mandibular Incisor. J Endod. 1997;23(8):479-84.

Recibido : 11-11-2011

Aceptado: 07-05-2012 